\title{
Cholinesterase inhibitors for co-morbid Alzhemer's disease dementia and schizophrenia: Systematic review and meta-analysis
}

\author{
Vellingiri Raja Badrakalimuthu
}

Parklands Hospital, Basingstoke, UK

Email: raja.badrakalimuthu@yahoo.co.uk

Received 17 February 2013; revised 20 March 2013; accepted 16 April 2013

Copyright (C 2013 Vellingiri Raja Badrakalimuthu. This is an open access article distributed under the Creative Commons Attribution License, which permits unrestricted use, distribution, and reproduction in any medium, provided the original work is properly cited.

\begin{abstract}
Cholinergic dysfunction is common to Alzheimer's dementia and Schizophrenia. The objective of this study is to undertake a systematic review and metaanalysis of the literature on the cognitive and clinical effects of cholinesterase inhibitors administered to patients with schizophrenia and co-morbid Alzheimer's disease dementia. A literature search of the MEDLINE, CINAHL, PubMed, Psycinfo, EMBASE, AMED, BNI, HMIC and Health Business Elite databases has been performed (up to January 2013) to investigate the use of cholinesterase inhibitors in patients with schizophrenia and dementia. The terms "schizophrenia”, “dementia”, "rivastigmine”, “donepezil", "galantamine" and "cognitive deficit" have been searched, with a restriction for English language. Five published studies including 1 RCT have been included for the qualitative review. Treatments include Donepezil 5 mg and $10 \mathrm{mg}$ as well as Rivastigmine $9 \mathrm{mg}$. The numbers of participants vary from 2 in case report to 20 in the RCT. Only 1 study qualifies for meta-analysis. There is a very limited evidence on the efficacy and safety of using acetylcholinesterase inhibitors in the management of dementia co-morbid with schizophrenia. The only randomised controlled study shows lack of evidence in terms of efficacy in using cholinesterase inhibitors in the management of dementia with schizophrenia. Future research projects will have to look at an adequate sample size to explore treatment on various cognitive and noncognitive domains and the sample should be selected by using definitive and internationally acceptable diagnostic criteria.
\end{abstract}

Keywords: Dementia; Schizophrenia; Acetyl Cholinesterase Inhibitors

\section{INTRODUCTION}

Comorbid schizophrenia and dementia are becoming an increasingly common clinical presentation with increaseing life expectancy in patients with schizophrenia and drive towards early diagnosis and management of dementia. It is estimated that up to two-thirds of elderly patients with chronic schizophrenia will meet the criteria for dementia [1].

Cholinergic dysfunction is pathophysiological to Alzheimer's disease dementia and cholinesterase inhibitors such as donepezil, rivastigmine and galantamine used in patients with Alzheimer's disease dementia provides beneficial effects on cognition, behavioural and psychological symptoms as well as activities of daily living [2]. Cognitive dysfunction has been recognised as a core feature of schizophrenia with deficits involving memory, attention, executive function, verbal fluency and motor skills, predicting a poor social outcome in patients with schizophrenia [3-5]. Cholinergic dysfunction is said to contribute to the pathophysiology of schizophrenia with reduced numbers of post-synaptic nicotinic receptors, and reduced numbers of muscarinic receptors in cortex and hippocampus associated with significant cognitive deficits in schizophrenia [6]. A systematic review and meta-analysis of using cholinesterase inhibitors in patients with schizophrenia and schizoaffective disorders concludes that specific cognitive deficits including memory, and the motor speed and attention part of executive function are responsive to rivastigmine, donepezil and galantamine as adjunctive therapy [7].

Considering the overlap in pathophysiology between dementia and schizophrenia and the increasing numbers of clinical presentations with co-morbid schizophrenia and Alzheimer's disease dementia, it is important to investigate the current understanding of the use of cholinesterase inhibitors in patients with dementia and 
schizophrenia.

The objective of this study is to undertake a systematic review and meta-analysis of the literature on the cognitive and clinical effects of cholinesterase inhibitors administered on patients with schizophrenia and co-morbid Alzheimer's disease dementia.

\section{METHODOLOGY}

This study is a systematic review and meta-analysis of the literature and the results of published trials that evaluated the use of cholinesterase inhibitors as treatment for patients with schizophrenia and co-morbid Alzheimer's disease dementia.

\subsection{Types of Interventions}

A cholinesterase inhibitor given at the dose recommended as optimal by the manufacturing pharmaceutical company (donepezil $10 \mathrm{mg} /$ day in one dose, galantamine $24 \mathrm{mg} /$ day in two doses, and rivastigmine $6-12 \mathrm{mg} /$ day in 2 doses).

\subsection{Data Sources}

A literature search of the MEDLINE, CINAHL, PubMed, PsycINFO, EMBASE, AMED, BNI, HMIC and Health Business Elite databases has been performed (up to January 2013) to investigate the use of cholinesterase inhibitors in patients with schizophrenia and dementia. The terms "schizophrenia", "dementia", "rivastigmine", "donepezil", "galantamine" and "cognitive deficit" have been searched, with a restriction for English language. Only original articles have been included. Additionally, the bibliographic data of all included publications for further studies and conference proceedings abstracts have been reviewed where available. Authors of trials have been contacted to obtain more information on particular aspects of trials. All articles that presented originnal data from randomized, double-blind, placebo-controlled, crossover and open-label trials as well as case reports with rivastigmine, donepezil or galantamine in patients with schizophrenia and dementia are included in the qualitative review.

\subsection{Meta-Analysis}

Each article identified by the literature search is reviewed and all studies fulfilling the inclusion criteria are selected for the meta-analysis.

\subsection{Inclusion Criteria}

Studies meeting the following criteria are included: 1) randomized, controlled trials (RCT) of the use of a cholinesterase inhibitor (rivastigmine, donepezil or galantamine) as a treatment for dementia in patients on treatment for schizophrenia; 2) cognitive/clinical assessment is performed using validated rating scales; 3 ) data available on group means and standard deviations for baseline and post-intervention cognitive/clinical tests.

\subsection{Exclusion Criteria}

Studies are excluded from meta-analysis for the following reasons: 1) non-randomised and open label clinical trials/case study/letter/correspondence/review; 2) animal study; 3) studies involving use of cholinesterase inhibitors as an adjunct for treating cognitive impairment that does not meet the criteria for dementia in patients with schizophrenia; 4) studies involving use of cholinesterase inhibitors as an adjunct for treating cognitive impairment in non-Alzheimer's disease type of dementia in patients with schizophrenia; 5) where complete data is not available from the published report or from contacting the lead author.

\subsection{Clinical and Outcome Measures}

Scores on Alzheimer's Disease Assessment Scale-cognitive subscale (ADAS-Cog), Mini-Mental State Examination (MMSE), Neuro-Psychiatric Interview (NPI) and Clinical Global Impression of Severity of Illness \& Improvement (CGI) scales is analysed as the outcome measures. These give a comprehensive opinion involving the domains of cognition, behavioural and psychological symptoms of dementia. Scores on the Positive and Negative Syndrome Scale [PANSS] (subscales: total, positive and negative) is analyzed for the impact of the cholinesterase inhibitors on schizophrenia in patients with dementia.

\subsection{Statistical Methods}

It was planned to express results for dichotomous data as risk ratios (RR) with 95\% confidence intervals (CI) and results for continuous outcomes as mean difference (MD) or standardized mean difference (SMD). It had been planned to use a fixed-effect approach unless there was substantial heterogeneity, in which case a random-effects approach would be used exploring potential causes of heterogeneity.

\section{RESULTS}

\subsection{Systematic Review of Studies}

Five published studies including 1 case report and 1 RCT have been included for the qualitative review (Table 1). The study by Djordjevic et al. [8] is an abstract from a poster presentation and no further data could be collected from the first author.

The number of participants varies from 2 in case report to 20 in the RCT. The duration of studies varies 
Table 1. Studies included in systematic review.

\begin{tabular}{|c|c|c|c|c|c|c|c|}
\hline Study & Type & Drug/Dose & $\begin{array}{l}\text { Inclusion } \\
\text { Criteria }\end{array}$ & Size & Duration & $\begin{array}{l}\text { Outcome } \\
\text { measures }\end{array}$ & Results \\
\hline Stryjer et al. (2002) [9] & $\begin{array}{l}\text { Case } \\
\text { report }\end{array}$ & $\begin{array}{l}\text { Donepezil } \\
5 \mathrm{mg}\end{array}$ & $\begin{array}{l}\text { Schizophrenia } \\
\text { Dementia }\end{array}$ & 3 & $\begin{array}{l}54 \text { days- } \\
3 \text { months }\end{array}$ & $\begin{array}{l}\text { MMSE } \\
\text { CGI }\end{array}$ & $\begin{array}{l}{\text { Case } 2^{*}}^{*} \\
\text { MMSE improved from } 15 \text { - } 22 \\
\text { CGI } 2 \\
\text { Case } 3 \\
\text { MMSE improved from } 16 \text { - } 25 \\
\text { CGI } 2\end{array}$ \\
\hline Stryjer et al. (2003) [11] & Single blind & $\begin{array}{l}\text { Donepezil } \\
5 \mathrm{mg}\end{array}$ & $\begin{array}{l}\text { Schizophrenia } \\
\text { Dementia }\end{array}$ & 6 & $\begin{array}{l}4 \text { weeks } \\
\text { with an } \\
\text { option for } \\
\text { continuing } \\
\text { indefinitely }\end{array}$ & $\begin{array}{l}\text { ADAS-Cog } \\
\text { MMSE } \\
\text { CGI } \\
\text { PANSS }\end{array}$ & $\begin{array}{c}\text { Significant improvement in } \\
\text { MMSE and CGI } \\
(\mathrm{p}<0.01) \text {, but not in ADAS-Cog } \\
(\mathrm{p}=0.26) \text { and PANSS }(\mathrm{p}=0.16)\end{array}$ \\
\hline $\begin{array}{l}\text { Mendelsohn et al. (2004) } \\
\text { [12] }\end{array}$ & Open label & $\begin{array}{l}\text { Rivastigmine } \\
9 \mathrm{mg}\end{array}$ & $\begin{array}{l}\text { Schizophrenia } \\
\text { Dementia }\end{array}$ & 13 & 12 weeks & $\begin{array}{l}\text { ADAS-Cog } \\
\text { MMSE } \\
\text { PANSS } \\
\text { ADL }^{* *}\end{array}$ & $\begin{array}{c}\text { Improvement in MMSE } \\
(\mathrm{p}<0.01), \text { ADAS-Cog } \\
(\mathrm{p}<0.001) \text {, ADL }(\mathrm{p}<0.01) \text { and } \\
\text { PANSS }(\mathrm{p}<0.01) .\end{array}$ \\
\hline Mazeh et al. (2006) [10] & $\begin{array}{l}\text { RCT with placebo } \\
\text { cross over }\end{array}$ & $\begin{array}{l}\text { Donepezil } 5 \mathrm{mg} \\
\text { titrated to } 10 \mathrm{mg} \\
\text { after first week }\end{array}$ & $\begin{array}{l}\text { Schizophrenia } \\
\text { Prominent decline } \\
\text { on ADAS-Cog }\end{array}$ & 20 & 12 weeks & $\begin{array}{l}\text { ADAS-Cog } \\
\text { CGI } \\
\text { PANSS }\end{array}$ & $\begin{array}{c}\text { No statistical differences in CGI } \\
(\mathrm{p}=0.37) \text {, PANSS } \\
(\mathrm{p}=0.71) \text { and ADAS-Cog } \\
(\mathrm{p}=0.86) \text { between two groups. }\end{array}$ \\
\hline Djordjevic et al. (2010) [8] & Observational & Rivastigmine & $\begin{array}{c}\text { Schizophrenia } \\
\text { MMSE less than } 24\end{array}$ & 11 & 60 days & $\begin{array}{l}\text { MMSE } \\
\text { NPI } \\
\text { BPRS }^{* * *}\end{array}$ & $\begin{array}{l}\text { Significant improvements in } \\
\text { cognition and reduction in } \\
\text { behaviouristic functioning. }\end{array}$ \\
\hline
\end{tabular}

("Case 1 has been excluded as this involved a patient with Parkinson's disease related dementia; **Activities of Daily Living Scale; ${ }^{* * *}$ Brief Psychiatric Rating Scale.)

from 4 weeks to 12 weeks. Case report by Stryjer et al. [9] does not discuss criteria used for diagnosis of schizophrenia or dementia. DSM-IV criteria has been used for the diagnosis of schizophrenia in studies by Mazeh et al. [10] and Stryjer et al. [11] and ICD-10 criteria has been used by Djordjevic et al. [8]. The widely accepted criteria of a MMSE score of less than 24 has been used as diagnostic criteria for dementia in studies by Djordjevic et al. [8], Stryjer et al. [11] and a prominent decline in ADAS-Cog has been used as criteria by Mazeh et al. [10]. Treatments included Donepezil 5mg and 10mg as well as Rivastigmine $9 \mathrm{mg}$. Various combinations of assessment tools have been used to assess progress from treatment as explained in Table 1.

Although studies by Djordjevic et al. [8] and Mendelsohn et al. [12] along with study and case report by Stryjer et al. $[9,11]$ indicate an improvement in MMSE, there is no significant improvement or no improvement at all on the ADAS-Cog from the studies by Mendelsohn et al. [12] and Mazeh et al. [10]. In terms of CGI, the RCT by Mazeh et al. [10] shows no statistically significant improvement whilst the single blind and case reports from Stryjer et al. $[9,11]$ show improvements on the CGI. Reviewing the impact of treatment on schizophrenia, it appears that on the PANSS there is a significant improvement in the open label study by Mendelsohn et al. [12] whilst there is no significant improvement in the RCT by Mazeh et al. [10] and single blind study by Stryjer et al.
[11].

In terms of adverse effects, in the single blind study by Stryjer et al. [11] and the RCT by Mazeh et al. [10], 1 patient has demonstrated to fatigue and weakness and agitation has been reported leading to discontinuation of donepezil by Mazeh et al. [10]. In the study by Stryjer et al. [11] donepezil has been discontinued due to fatigue whiles in Mazeh et al.'s study [10], fatigue has undergone spontaneous remission.

\subsection{Meta Analysis}

Of the 5 studies from the systematic search, only the study by Mazeh et al. [10] is an RCT and hence meeting the criteria for inclusion in meta-analysis. This study shows a modest treatment effect for both placebo and donepezil treatment but no statistical differences were demonstrated between the two treatment groups (CGI p $=0.37$, PANSS $p=0.71$, ADAS-Cog $p=0.86$ ). Further analysis has not been carried out due to small sample size and lack of similar randomised controlled trials.

\section{DISCUSSION}

\subsection{Summary of Findings}

There is very limited evidence on the efficacy and safety of using acetylcholinesterase inhibitors in the management of dementia co-morbid with schizophrenia. The only RCT by Mazeh et al. shows lack of evidence in terms of efficacy in using cholinesterase inhibitors in the 
management of dementia with schizophrenia.

\subsection{Implications of Findings}

Alzheimer's dementia and Schizophrenia are complex disorders with involvement of multiple neurotransmitters and cortico-striato-thalamic circuit leading to many different types of symptoms. Although a cholinergic under activity has been hypothesised to be common to both these disorders, cholinergic dysfunction may not be causing all the symptoms and therefore cholinergic drugs such as acetylcholinesterase inhibitors, may not be showing significant improvement involving all domains. This is perhaps why the meta-analysis of cholinesterase inhibitors in treating cognitive impairment in schizophrenia [7] showed significant impact on selecting cognitive domains such as executive dysfunction and why this meta-analysis of cholinesterase inhibitors in dementia comorbid with schizophrenia fails to provide robust evidence on using these drugs in treating this presentation.

However, with the aging population and the longevity of patients with schizophrenia on antipsychotic medications, comorbid dementia and schizophrenia will start to present more frequently in psychogeriatric clinics. Hence there is a need to explore potentially beneficial treatments such as using cholinesterase inhibitors. Future research projects will have to look at an adequate sample size to explore treatment on various cognitive and noncognitive domains and the sample should be selected by using definitive and internationally acceptable diagnostic criteria.

\subsection{Methodological Limitations}

This is the first meta-analysis of use of cholinesterase inhibitors in schizophrenia comorbid with dementia. This study is limited for the lack of RCTs exploring the question. The studies included in this systematic review and meta-analysis are limited by the criteria used for diagnosis dementia in schizophrenia, lack of adequate sample size, lack of methodological rigor and having adequate follow-up.

\section{CONCLUSION}

There is very limited evidence on the efficacy and safety of using acetylcholineseterase inhibitors in the management of dementia co-morbid with schizophrenia. It is important to investigate the current understanding of the use of cholinesterase inhibitors in patients with dementia and schizophrenia through adequately powered randomised controlled trials.

\section{ACKNOWLEDGEMENT}

Ms. Marion Taylor, Librarian, Southern Health NHS Foundation Trust
Healthcare Library, Tom Rudd Unit, Moorgreen Hospital, Southampton.

\section{REFERENCES}

[1] Arnold, S., Gur, R., Shapiro, R., et al., (1995) Prospective clinicopathologic studies of schizophrenia: Accrual and assessment of patients. American Journal of Psychiatry, 152, 731-737.

[2] Raina, P., Santaguida, P., Ismaila, A., et al. (2008) Effectiveness of cholinesterase inhibitors and memantine for treating dementia: Evidence review for a clinical practice guideline. Annals of Internal Medicine, 148, 379-397. doi:10.7326/0003-4819-148-5-200803040-00009

[3] Aleman, A., Hijiman, R., De Haa, E.H.F., et al. (1999) Memory impairment in schizophrenia: A meta-analysis. American Journal of Psychiatry, 156, 1358-1366.

[4] Heinrichs, R.W. and Zakzanis, K.K. (1998) Neurocognitive deficits in schizophrenia: A quantitative review of the evidence. Neuropsychology, 12, 426-445. doi:10.1037/0894-4105.12.3.426

[5] Bokat, C.E. and Goldberg, T.E. (2003) Letter and category fluency in schizophrenic patients: A meta-analysis. Schizophrenia Research, 64, 73-78. doi:10.1016/S0920-9964(02)00282-7

[6] Lenzi, A. and Tuscano, D. (2004) Do acetylcholinesterase inhibitors have a role in improving cognitive impairment in patients with schizophrenia. International Journal of Pharmacological Medicine, 18, 139-148. doi:10.2165/00124363-200418030-00002

[7] Ribeiz, S., Bassitt, D., Arrais, J., et al. (2010) Cholinesterase inhibitors as adjunctive therapy in patients with schizophrenia and schizoaffective disorder: A systematic review and meta-analysis of the literature. CNS Drugs, 24, 303-317. doi:10.2165/11530260-000000000-00000

[8] Djordjevic, S., Japalak, S. and Vodojevic, V. (2010) Effect of rivastigmine tartarat in therapy of patients with schizophrenia. Annals of General Psychiatry, 9, S91.

[9] Stryjer, R., Bar, F., Strous, R., et al. (2002) Donepezil management of schizophrenia associated with dementia. Journal of Clinical Psychopharmacology, 22, 226-229. doi:10.1097/00004714-200204000-00021

[10] D. Mazeh, H. Zemishlani, Y. Braka, et al. (2006) Donepezil for the negative signs in elderly patients with schizophrenia: An add-on, double blind, crossover, placebo-controlled study. International Journal of Psychogeriatrics, 18, 429-436. doi:10.1017/S1041610205003017

[11] Stryjer, R., Strous, R., Bar, F., et al. (2003) Beneficial effects of donepezil augmentation for the management of comorbid schizophrenia and dementia. Clinical Neuropharmacology, 26, 12-17. doi:10.1097/00002826-200301000-00004

[12] Mendelsohn, E., Rosenthal, M., Bohiri, Y., et al. (2004) Rivastigmine augmentation in the management of chronic schizophrenia with comorbid dementia: An open-label study investigating effects on cognition, behaviour and activities of daily living. International Journal of Clinical Pharmacology, 19, 319-324. 